The feedback from all candidates has been very positive so far (figures 1, 2, 3, and 4). We have improved confidence levels and most candidates are currently satisfied with the current PACES teaching structure at EDGH. In addition, most candidates found that supervised bedside practice is one of the top two useful methods to preparing for PACES. There is room for improvement taken from the feedback in that we could involve more senior members (consultants and registrars) in providing the bedside sessions and to have more stations 2 and 4 (history taking and communication skills) practice sessions.

\section{MAINTAINING ESSENTIAL PHYSICAL HEALTH CHECKS IN AN ACUTE PSYCHIATRIC HOSPITAL}

Rohan Malik*, Michael Ayres. Bradford District Care Foundation Trust

\subsection{6/leader-2018-FMLM.104}

\section{Summary}

- The RCPsych advises ECGs on newly admitted patients.

- ECGs were not performed within the recommended timeframe. This negatively impacted patient safety.

- Introduction of a three times weekly 'board round' check increased compliance with the standard.

- ECG machine availability is recognized as one factor in failing to adhere to the $48 \mathrm{~h}$ standard.

Background The RCPsych published integrated physical health guidelines for newly admitted inpatients, outlining the need to 'arrange physical health checks within $48 \mathrm{~h}$ ' including ECGs to exclude cardiac side effects of psychotropic medication. ${ }^{1}$

Method A baseline audit conducted in December 2016 showed that only a small proportion (33\%) of patients had an ECG performed within 48 hours of admission. A $3 \times$ weekly 'Physical Health Board Round' was introduced on three wards to see if this improved compliance. A re-audit was undertaken in May 2017 to assess if there had been any improvement in the frequency of performance and documentation of ECGs.

Results After the intervention, ECGs were performed within 48 hours of admission in 45 cases and not in 68 cases. The most common reason an ECG was not performed was that the patient declined $(n=23)$. The compliance therefore increased to $45 / 90=50 \%$

Conclusion Implementing $3 \times$ weekly physical health 'board round' improves ECG compliance. $50 \%$ of patients are still not receiving ECGs, some with no explanation. Further work should be undertaken to explore this deficit. Investment has been made into further ECG machines for the hospital as a result of this study.

\section{REFERENCE}

1. Royal College of Psychiatrists. Integrated physical health pathway 2014. Available at https://www.rcpsych.ac.uk/pdf/NAS\%2OIntegrated\%20Physical\%20Health\% 20Pathway\%20Dec\%2012.pdf [accessed: 25/10/17].

\section{E-handover: Passing the electric baton to enhance communication}

\section{HANDOVER AND COMMUNICATION}

Orod Osanlou, Michael Fisher, Pam Stanford, Joseph Abbott, Eni Ann Riley, Hon-wing Shek, Andrew Davies, Tony Morley, Gemma Jones, Mike Beadsworth, Julian Hobbs, Rostam Osanlou. Royal Liverpool and Broadgreen University Hospitals NHS Trust

\subsection{6/leader-2018-FMLM.105}

Introduction Handover is a dangerous time in a patient's journey. Sub-optimal communication can lead to confusion, ambiguity, delays in treatment, medication errors and foster a culture of a lack of accountability.

Aim An electronic handover system (e-handover) was developed with the aim of enhancing communication and improving patient safety through creating an electronic, auditable trail of all handover between doctors and nurses.

Method Following stakeholder engagement (including patients), bespoke e-handover software was designed "in-house" that intelligently links with other trust electronic databases used for documenting in patient notes, admitting, referring and discharging patients. Clinicians input patient tasks to be completed (via SBAR system or equivalent) into e-handover both in hours and out of hours. Handovers are triaged and delegated to appropriate "on call" clinicians who have been provided with tablets that can access e-handover remotely.

Implementation Following a successful pilot, it has rolled out to all 48 level one wards in the trust with over 1,000 staff members trained. It has since been expanded to include communication with pharmacists.

Results Compared with the previous system of handover, the quality of SBAR style handover has improved by over $60 \%$ and the percent of illegible handovers has fallen from $9.3 \%$ to $0 \%$. The documented names of staff uploading and signing off tasks have increased from $33.5 \%$ and $32.1 \%$ respectively to $100 \%$, and the documented time that jobs have been completed has improved from $0 \%$ to $89.4 \%$. The volume of jobs on e-handover has also fallen by $42 \%$ compared with the previous "red book" system, allowing staff more time to focus on unwell patients and patient flow. 92\% of staff $(n=81)$ feel that e-handover is user friendly and 91\% would recommend it. Importantly, $71 \%$ feel that handovers are safer and $61 \%$ feel that patient safety has improved as a result of e-handover. The mean number of cardiac arrests as calculated by t-test has also fallen since introduction of e-handover $(p=0.032)$, although causality cannot be determined due to other electronic systems for sepsis and recording resuscitation status having also been introduced on a similar timescale.

Conclusion E-handover is a user friendly and effective method to improve the quality and accountability of handover, improving patient safety and is easily scalable throughout the whole NHS. 